\title{
Cost comparison of different Translucent Optical Network Architectures
}

\author{
Maarten De Groote ${ }^{1}$, Konstantinos Manousakis ${ }^{2}$, Panagiotis Kokkinos ${ }^{2}$, Didier Colle ${ }^{1}$, Mario \\ Pickavet $^{1}$, Konstantinos Christodoulopoulos ${ }^{2}$, Emmanouel (Manos) Varvarigos ${ }^{2}$, Piet Demeester ${ }^{1}$ \\ ${ }^{1}$ Department of Information Technology (INTEC), Ghent University - IBBT, \\ Gaston Crommenlaan 8, B-9050, Gent, Belgium \\ E-mail: \{maarten.degroote\}@intec.ugent.be \\ ${ }^{2}$ Research Academic Computer Technology Institute, Patras, Greece \\ E-mail: \{manousak\}@ ceid.upatras.gr
}

\begin{abstract}
Ever-increasing bandwidth demands and higher flexibility are the main challenges for the next generation optical core networks. A new trend in order to address these challenges is to consider the impairments of the lightpaths during the design of optical networks. In our work, we focus on translucent optical networks, where some lightpaths are routed transparently, whereas others go through a number of regenerators. We present a cost analysis of design strategies, which are based either on an exact Quality of Transmission (QoT) validation or on a relaxed one and attempt to reduce the amount of regenerators used. In the exact design strategy, regenerators are required if the QoT of a candidate lightpath is below a predefined threshold, assuming empty network conditions. In the relaxed strategy, this predefined threshold is lower, while it is assumed that the network is fully loaded. We evaluate techno-economically the suggested design solutions and also show that adding more flexibility to the optical nodes has a large impact to the total infrastructure cost.
\end{abstract}

Index Terms- next-generation optical core, network dimensioning, cost analysis, routing and wavelength assignment, physical layer impairments

\section{INTRODUCTION}

With growing high-bandwidth demands, caused by fiber to the home, video on demand, high definition multimedia, etc., next-generation optical core networks will require significant improvements in capacity and configurability. These advancements need to be achieved with new technologies that are scalable with respect to network cost, size, and power requirements.

In opaque networks the signal is regenerated at every intermediate node along a lightpath via optical-electronicoptical (OEO) conversion. The network cost could be reduced by employing regenerators only at specific nodes of the network. When regenerators are available, a lengthy end-toend connection that needs regeneration at some intermediate node(s) is set up in a multi-segment manner so that it is served by two or more consecutive transparent lightpath segments. Optical networks, where some lightpaths are routed transparently, while others go through a number of regenerators, are known as translucent optical networks. In some networks it is also feasible for the data signal to remain in the optical domain for the entire path and these networks are known as transparent networks.

In transparent and translucent networks, it is important to propose algorithms that select the routes for the connection requests and the wavelengths that will be used on each of the links along these routes, so as to optimize certain desired performance metrics. This is known as the routing and wavelength assignment (RWA) problem. An offline RWA algorithm is executed when the network is initially set up for network provisioning, and is also executed periodically, or when traffic changes substantially.

The typical objectives of this problem are to reduce both the blocking ratio over an infinite time horizon and the network cost in terms of Capital Expenditure (CapEx) and Operational Expenditure (OpEx).

The main contribution of the paper is the cost analysis and comparison of two alternative strategies for provisioning an optical network, namely the exact and the relaxed one. In this way, a network designer will be able to plan the network efficiently, considering both performance and cost related criteria. In particular, these two approaches are compared in the basis of a techno-economic analysis that takes into account both CapEx (optical cross - connect (OXC), regenerator and other equipment costs) and network related OpEx (power consumption, floor space, repair costs) considerations. To make it more concrete several node architectures are considered in this paper. All types can remotely configure the transit traffic and differ in type of add/drop features. The impact of the different configurations on the total cost will also be evaluated.

The rest of the paper is organized as follows. In Section II, we explain the physical impairments present in optical networks. Section III describes the exact and relaxed design strategies that account for the physical impairments. The node architectures considered and the cost model used in the techno-economic evaluation of the suggested strategies is described in Sections IV and V respectively. Section VI 
presents the evaluation of the design strategies in terms of regenerators, wavelengths utilized and CapEx, OpEx. Finally, conclusions and directions for future work are given in Section VII.

\section{Physical ImPairments AND QUality OF TRANSMISSION}

In transparent or translucent optical networks a connection blocking may occur (i) due to the unavailability of free wavelengths or links (network-layer blocking) and (ii) due to the physical layer impairments, introduced by the non-ideal physical layer, which may degrade the signal quality to the extent that the lightpath is infeasible (physical-layer blocking). Several criteria can be used to evaluate the signal quality of a lightpath. Among a number of measurable optical transmission quality attributes, the Q-factor seems to be more suitable as a metric to be integrated in an RWA algorithm, because of its immediate relation to the bit error rate (BER), assuming a Gaussian shaped noise. The Q-factor is the electrical signal-tonoise ratio at the input of the decision circuit in the receiver's terminal [2][3]. When considering Impairment Aware -RWA (IA-RWA) algorithms, it is useful to categorize the physical layer impairments (PLIs) to those that affect the same lightpath [4], to be referred to as Class 1 impairments [Polarization Mode Dispersion (PMD), Chromatic Dispersion (CD), Filter concatenation (FC), Self-Phase Modulation (SPM)], and to those that are generated by the interference among lightpaths, to be referred to as Class 2 impairments [Crosstalk (XT), Cross-Phase Modulation (XPM), Four Wave Mixing (FWM)].

\section{EXACT VERSUS RELAXED DESIGN OF TRANSLUCENT NETWORKS IN THE PRESENCE OF PLIS}

In this section we focus on translucent optical networks and examine offline IA-RWA algorithms. In translucent optical networks, regenerators are employed at some but not all the network nodes. Some of the connections established are routed transparently, while others, typically those served by lengthy paths, may need to utilize several of the available regenerators to restore the transmitted signal's quality. Offline IA-RWA algorithms are employed in translucent networks; these algorithms decide on the lightpaths to be established, select the regeneration sites and the number of regenerators that need to be deployed on these sites, so as to serve a given set of connection requests (traffic matrix).

In order to provision the network we compare two different approaches, the exact and the relaxed approach, which are based on the same IA-RWA algorithm presented in [5], consisting of three phases. In the first phase, see Fig. 1, the connection demands are distinguished into those that can be served transparently and those that have to be served using regenerators. In the exact design strategy the quality of transmission (QoT) of a candidate lightpath, serving a connection request, is evaluated against a predefined threshold and assuming empty network conditions. In this way only the Class 1 impairments affecting the QoT of the paths are considered. However, in the following phases of the exact approach both Class 1 and Class 2 impairments are taken into account. On the other hand in the relaxed strategy the QoT threshold is lower, and specifically equal to the threshold of

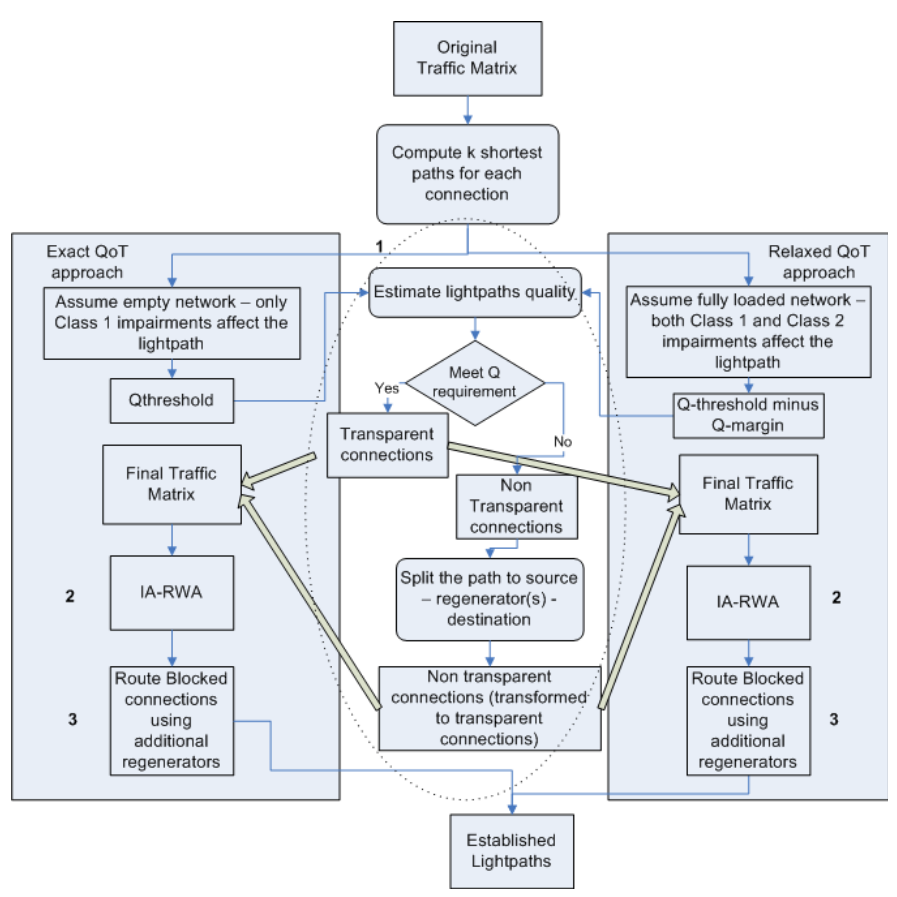

Fig. 1: Flow chart of the exact versus relaxed design approaches, with the three main phases.

the exact strategy minus a predefined Q-margin. In addition in the relaxed strategy the QoT of each candidate lightpath is calculated assuming that all wavelengths on all the links it uses are occupied. In this way both Class 1 and Class 2 impairments affecting the QoT of the paths are considered. Also, this assumption results in the over-provisioning of the network, as exhibited by the number of regenerators used. In both approaches a quality of transmission evaluation estimator module (Q-Tool) developed within the DICONET project [6] is used for assessing the QoT of lightpaths.

Next, the non-transparent connections are transformed into a sequence of transparent connections by routing them through a series of regenerators. To do so, the algorithm formulates a virtual topology problem. The virtual topology consists of the original network's regeneration sites, with (virtual) links between any pair of transparently connected regeneration sites. Each virtual link of the paths chosen in the virtual topology to serve a connection corresponds to a transparent sub-path (lightpath) in the physical topology. The algorithms used for routing the non-transparent traffic demands in the virtual topology, are based on a $k$-shortest path algorithm, with the cost of a virtual link defined as the number of physical links (physical hops) it consists of. With this definition, the optimal virtual path is the one that traverses the minimum number of physical nodes.

Then the algorithm selects the routes to be followed by nontransparent connections by minimizing one of the following: i) the maximum number of regenerators used among all network nodes (max algorithm), or ii) the total number of regenerators used in the network (sum algorithm), or iii) the number of regeneration sites (sites algorithm). To perform this optimization, the virtual topology problem is formulated as an integer linear program (ILP). At the end of the first phase the initial traffic matrix has been transformed into a new traffic 
matrix whose source-destination pairs can, in principle, be transparently connected.

In the second phase, an IA-RWA algorithm for transparent networks is applied, with input from the transformed transparent traffic matrix, in order to select the routes and wavelengths to be used. The IA-RWA algorithm we use in our simulation experiments is the one proposed in [7], which is based on a ILP relaxation formulation. Both Class 1 and Class 2 impairments are handled by this algorithm. Finally, in the third phase, the connections that were rejected in the second phase due to physical-layer blocking are rerouted through new regenerators.

\section{NODE ARCHITECTURES}

Up to now the described algorithms assume a node design without any architectural limitations. Next, we present three node architectures for the optical cross-connects (OXCs); their characteristics affect the final cost of the design strategy applied for serving a set of connection requests. All node architectures offer the same functionality for the transit traffic, full remote re-configurability. They primarily differ in how the traffic local to the node is treated. Therefore, categorizing OXCs mainly refers to the features/flexibility of add/drop ports.

\section{A. Optical add/drop ports}

First, we discuss different optical add/drop port types, that will then be used in the different OXCs, more technical information can be found in [8].

\section{1) Colored-colorless ports}

Currently optical networks are evolving from colored to colorless add/drop ports which, unlike colored add/drop ports, do not have a permanently assigned wavelength channel but rather are provisioned as to which wavelength channel will be added/dropped. Considering tunable transponders (TSP), colorless ports allow the wavelength to be selected and provisioned remotely. Colorless ports are generally created by replacing a fixed wavelength demultiplexing element (for example an arrayed waveguide grating (AWG) component) with a wavelength selective switch (WSS). A WSS can steer each optical channel present on its input port toward one of its output ports.

\section{2) Directionless ports}

If a node is equipped with directed add/drop ports, a channel on a specific transmission fiber entering the node can be dropped only by a demultiplexing element connected to this transmission fiber. In the same way, a locally added channel leaves the node on a pre-defined transmission fiber depending on the selected add port. Thus in an OXC with directionless add/drop ports not only transit traffic is switched from/to arbitrary transmission fibers, local traffic can be switched flexibly, too. Typically this directionless port property is realized by re-dedicating a transmission fiber port to a local port. It should be noted that for this realization a particular wavelength can still be added/dropped to only one transmission fiber port. The use of a particular wavelength, blocks adding/dropping of the same wavelength to another fiber port.

\section{3) Partly blocking-free ports}

An intermediate architectural step between fully wavelength blocking-free ports irrespective of the number of locally identical transponder wavelengths on one side and a single add/drop port for each wavelength on the other side should be mentioned here. This could earn high practical relevance in future optical networks as it allows an easy scaling of the number of wavelength blocking-free ports. The basic idea is to re-dedicate multiple transmission fiber ports (equipped with e.g. WSS and power splitter) as local ports.

\section{B. Optical cross-Connects}

In the next subsections, we describe the three considered node implementations. These are the fixed colored OXC, the colorless directionless non-blocking free $\mathrm{OXC}$ and the colorless directionless blocking free OXC.

\section{1) Fixed colored Optical cross-Connect (OXC) (node 1)}

The colored OXC can remotely configure all transit with a broadcast and select architecture. The incoming channels are broadcasted (with a splitter) to all other network interfaces (for example boxes B and C in Fig. 3). A WSS is connected to the outgoing fiber and can select which wavelength from which other network interface or add/drop terminal it wants to add. In this node architecture, fixed and direction-specific transponders, the XPDRs in Fig. 2, are used. This means each transponder is connected through an AWG component. If a particular wavelength is not equipped in an add/drop terminal, it cannot be used to add/drop at that particular network interface. The advantage is that there is no need for extra WSS equipment in the add/drop terminals.

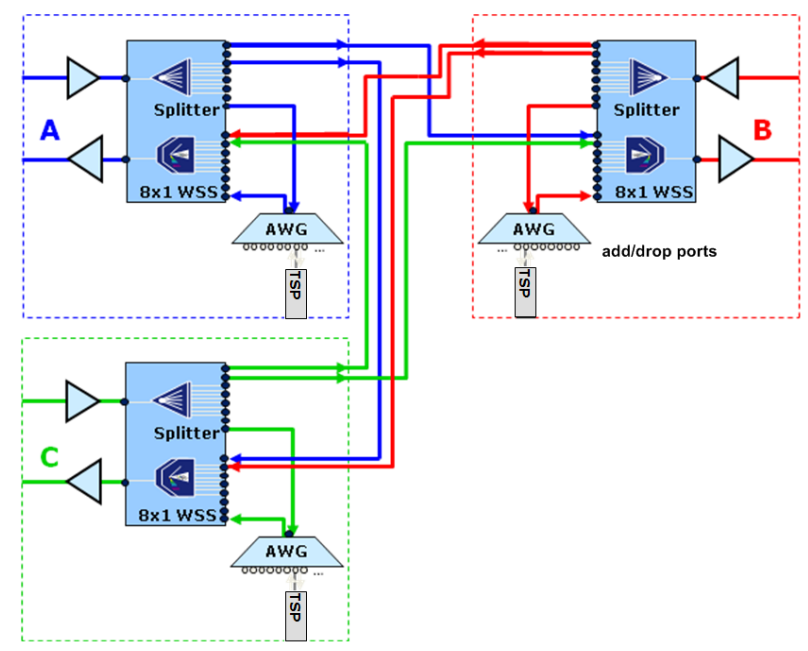

Fig. 2: A fixed colored Optical cross-Connect (OXC). Remote reconfigurability for transit traffic and static add/drop for local traffic. 
2) Colorless directionless non-blocking free OXC (node2)

To add more flexibility at the add/drop side, it is possible to use an extra network interface instead of an AWG component to select which wavelengths have to be dropped from the other network interfaces. This extra network interface adds the directionless feature of the node (see Fig. 3), and is the first stage of the add/drop terminal. The constraint of this configuration is that only one unique wavelength can be dropped at an add/drop terminal, because a WSS can only drop the same wavelength channel once to its output port. The colorless feature is implemented by the second stage of the add/drop terminal. In that box a combiner is used to add all the wavelengths and a WSS is used to select which wavelength you want to drop at which port. In this node architecture we consider only one add/drop terminal this would result in the best cost optimized node architecture. So every wavelength can only be dropped once for this reason it is called nonblocking fee.

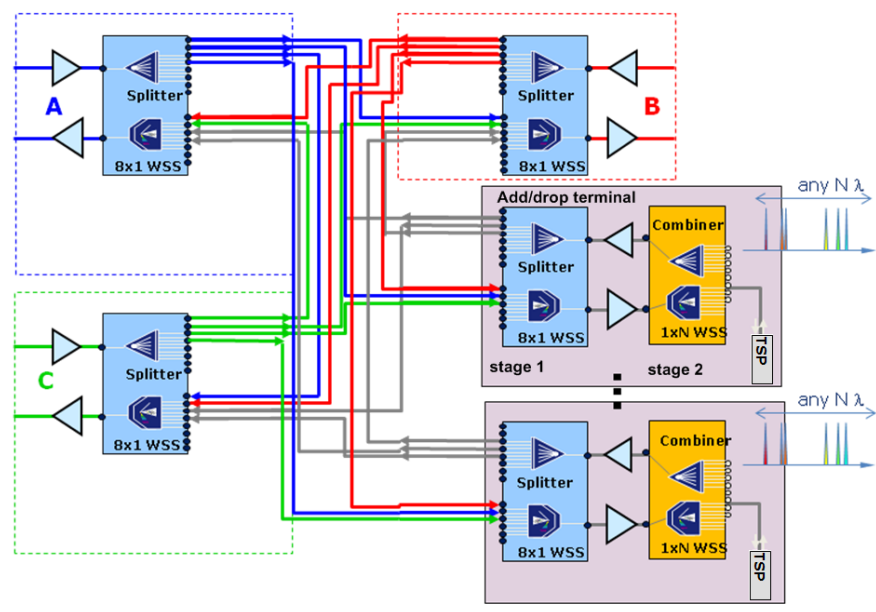

Fig. 3: Colorless directionless non-blocking free OXC. Here the number of add/drop terminals will be less than the number of network interfaces. For node 2 we took 1 add/drop terminal

\section{3) Colorless directionless blocking free OXC (node 3)}

For this node architecture the same number of add/drop terminals as the degree of the node is used. This would be the same architectures as in Fig. 3 but with 3 add/drop terminals for a node with degree 3 . This consideration will prevent a wavelength blocking at the add/drop terminal. More add/drop terminals will translate in a higher cost of the node. Because there are more network interfaces necessary and the number of ports has to be bigger.

\section{COST MODEL}

In this section we present a detailed description of the costs, consisting of CapEx and network related OpEx, used in our work for the techno-economic evaluation of the two optical design strategies presented in Section III. The cost model considered is based on actual list prices of all the important components. The model is an extension on the model described in [9]. After the calculation of the relative costs, with a transponder as base, all values were checked by different system vendors and network operators, see Table I.

Costs are modeled with different levels of detail; using driver based and dedicated cost modeling. More information on the different methods can be found in [10].

\section{A. Capital expenditures}

A summary of all the used costs in the model are shown in Table I. Extra necessary figures such as power and failure rates are included too. The model to calculate the network related OpEx will make use of these.

The cost of the different node architectures is based on their physical implementation, see Section IV. An important subpart is the network interface (NI); its cost $\left(C_{N I}\right)$ is based on the cost of the WSS $\left(C_{W S S}\right)$, the number of necessary splitter ports $\left(C_{\text {splitter }}\right)$ and a fixed cost for the casing $\left(C_{\text {case_NI }}\right)$ (Equation $1)$.

TABLE I

COST MODEL COMPONENTS

\begin{tabular}{|c|c|c|c|}
\hline Equipment & $\begin{array}{c}\text { Relative } \\
\text { cost }\end{array}$ & $\begin{array}{c}\text { Power } \\
\text { consumption } \\
{[\mathrm{W}]}\end{array}$ & MTBF \\
\hline Long reach $10 \mathrm{G}$ TSP & 1 & 30 & 250,000 \\
\hline EDFA, double stages & 1.33 & 25 & 500,000 \\
\hline EDFA, single stage & 1 & 15 & 500,000 \\
\hline 1x4WSS (80 channels) & 2.35 & 30 & 300,000 \\
\hline $1 \times 8$ WSS & 4.7 & 40 & 300,000 \\
\hline $1 \times 20$ WSS & 7.05 & 50 & 300,000 \\
\hline $1 \times 40 \mathrm{WSS}$ & 10.58 & 60 & $150,000^{2}$ \\
\hline Splitter $^{1}$ & 0.05 & - & - \\
\hline Combiner $^{1}$ & 0.13 & - & - \\
\hline AWG & 0.7 & - & - \\
\hline Regenerator & 1 & 30 & 250,000 \\
\hline Casing node & 2.5 & - & - \\
\hline Casing network interface & $1-4$ & $2-8$ & - \\
\hline Casing AWG & 0.5 & - & - \\
\hline
\end{tabular}

Transponder (TSP) is bidirectional considered, all other components are unidirectional.

MTBF (Mean time between failures)

EDFA (Erbium Doped Fiber Amplifier)

${ }^{1}$ Cost per port

${ }^{2}$ considered here 2 devices for the MTBF

Equation 2 shows the total cost for node $1\left(C_{\text {node }}\right)$, see section IV. The degree $N$ of a node is the most determining parameter of the total node cost. In a colored add/drop terminal there is also a fixed cost included. In addition, per node there is a fixed cost to represent the rack and other fixed equipment $\left(C_{\text {case_node }}\right)$.

$$
\begin{aligned}
& C_{N I}=C_{W S S}+C_{\text {splitter }}+C_{\text {case_NI }} \\
& C_{\text {node_ } 1}=N \cdot\left(C_{N I}+C_{A W G}+2 \cdot C_{E D F A 2}\right)+C_{\text {case_node }}
\end{aligned}
$$

$C_{A W G}=$ cost $\mathrm{AWG}$ and casing $\mathrm{AWG}$

$C_{E D F A 2}=$ cost of a double stage EDFA 
Equation 3 shows the total cost for node 2 and node 3 $\left(C_{\text {node_ } 2 \mid 3}\right)$, see section IV. We include an extra parameter, the number of add/drop terminals $(R)$. An add/drop terminal contains one subpart like a network interface and one with a combiner instead of a splitter. This last part will vary in relation to the number of added channels. To compensate the extra signal degradations, a single stage EDFA is included in both directions.

$$
\begin{aligned}
C_{\text {node_ } 2 \mid 3}= & N \cdot\left(C_{N I}+2 \cdot C_{E D F A 2}\right)+ \\
& R \cdot\left(C_{a / d_{-} 1}+C_{a / d_{-} 2}+2 \cdot C_{E D F A 1}\right)+C_{\text {case_node }}
\end{aligned}
$$

$C_{a / d_{-} l}=$ cost of stage 1 of add/drop terminal similar to $C_{N I}$ $C_{a / d \_2}=$ cost of stage 2 of add/drop terminal similar to $C_{N I}$

\section{B. Network related OpEx}

Network related OpEx are yearly recurring costs closely related to the total infrastructure cost. In the same way we dimension the nodes, it is also possible to calculate the total power consumption of all the nodes. All used input values are shown in Table II.

\section{Power consumption}

The inputs are the power of a WSS, the fixed power per network interface and the fixed power per add/drop terminal. In the total power consumption, we will also include the power of the transponders and the EDFAs. We assume a reference price of $0.1 €$ for $1 \mathrm{KW}$.

\section{Floor space}

As input we define the number of slots per WSS in the network interface and the WSS in the add/drop terminal. In the same way that the cost of the node is calculated, it is possible to calculate the total number of slots. In the Colored case, the number of slots per AWG component is needed too.

In the rent cost of the floor space we also include the general maintenance of the racks. This results in a higher cost per square meter than the actual renting cost. We consider here a cost of $50 €$ per square meter per month [11].

\section{Repair costs}

If the Mean time between failures (MTBF) and the number of active components are known, then we can calculate the number of failures in the whole network. In the cost per failure we include the mean repair cost (see Table II) and the cost of the equipment.

$$
M T B F=\frac{T}{R}
$$

$\mathrm{T}=$ total time

$\mathrm{R}=$ number of failurs
TABLE II

NETWORK OPEX COST MODEL

\begin{tabular}{ll}
\hline \hline Network OpEx input & Relative cost \\
\hline Cost per square meter per year & 0.27 \\
Cost per kW per year & 0.79 \\
& 0.2 \\
Repair cost* per WSS failure & 0.1 \\
Repair cost* per Transponder failure & 0.1 \\
& 0.1 \\
Repair cost* EDFA, double stage & \\
Repair cost* EDFA, single stage & \\
*The repair cost is without the equipment. In the total repair cost the \\
necessary equipment will be included.
\end{tabular}

\section{EVALUATION}

\section{A. Performance evaluation}

We carried out a number of simulation experiments, evaluating the performance of the previously described offline IA-RWA algorithms for translucent networks. The network topology used in our simulations was the Geant-2 network, which is a translucent Pan-European network with 34 nodes and 108 directional links. The traffic matrix is based on the traffic of 2009 and consists of a total of 826 connections. The initial traffic matrix is symmetric, but the traffic is not necessary routed bidirectional, same route in both directions. All single-hop connections were able to be served transparently, but some multi-hop connections were not, making the use of regenerators necessary for some connections. We assumed that the number of regeneration sites is not restricted; that is, every node in the network is capable

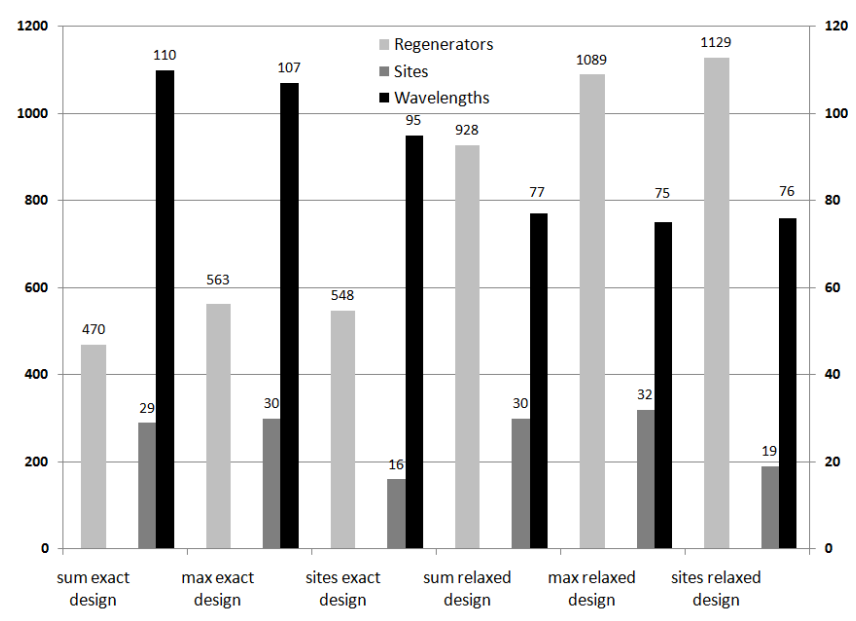

Fig. 4: Total number of regenerators, total number of regeneration sites and minimum number of wavelengths for 826 connection demands and unrestricted regeneration sites.

of accommodating regenerators. It was up to the IA-RWA algorithms to solve the regeneration placement problem, in order to decide the regeneration sites and the number of regenerators to deploy on each site. The Q-factor threshold was taken equal to $15.5 \mathrm{~dB}$ and the predefined Q-margin for the relaxed approach was equal to $0.5 \mathrm{~dB}$.

In Fig. 4 we graph the total number of regenerators, the total number of regeneration sites, and the minimum number of 
wavelengths required in the network, to reach zero blocking. The performance of each algorithm is closely related to the metric it minimizes. As depicted in Fig. 4 the IA-RWA algorithms for translucent networks that are based on the exact approach exhibit better performance when compared to the algorithms that are based on the relaxed approach and provision the network under the full network load assumption. Using this assumption more regenerators (at least twice and in many cases even more) are needed in order to satisfy the same demand matrix. The difference in the required number of regenerators can be explained as follows. Algorithms under the full network load assumption overuse the available resources in order to minimize the physical layer blocking. On the other hand, using the exact approach better use of the network resources, in terms of regenerators, is made.

Another performance metric, see Fig.4, is the minimum number of required wavelengths in the network. Some components in the network or wavelength specific and complexity will increase with higher number of wavelengths. The results of the RWA show in general an increase in wavelengths in the exact design approach. Based on these results, it becomes not evident anymore to point a clear winner. For this reason we perform a more detailed technoeconomic evaluation of both approaches.

\section{B. Techno- economic evaluation}

In this subsection we perform the techno-economic evaluation of the exact versus relaxed approaches, using as baseline the traffic matrix of 2009 (with 826 10G demands). Also, in order to check the costs' evolution the following years (e.g. the year 2011), we calculated the corresponding costs increasing by $50 \%$ every year the traffic matrix (in Gbps demands) of 2009. The resulted 2011 traffic matrix contains 1018 demands. Due to practical limitations we consider 80 wavelengths per fiber, if that is not sufficient an extra fiber link is added. Another topic we evaluate in this subsection is the impact of the node architectures on the total cost of the infrastructure.

\section{1) CapEx evaluation}

We calculated the total cost of the infrastructure, using the outputs of the two offline IA-RWA algorithms described in Section III. The cost of the transponders can increase between $35 \%$ and $48 \%$, if we compare an exact design approach versus the relaxed one, see Fig. 5. This would result in a cost benefit of the total system, if the other costs of components are the same for both cases, but this is not the case. We can also observe that the cost for the network interfaces is increased by $116 \%$ between "sum relaxed design 2009" and "sum exact design 2009". This is caused by the increased number of wavelengths needed in the network that results in extra fibers between several nodes, and a higher node cost because the degree determines the total node cost. Only the exact design approach with sites optimization is cheaper than its relaxed variant. This is due to the reduced cost of the network interfaces in the exact approach, making the benefits of the savings in transponders more clear. If we add more connections, there is a high increase of cost because of the extra fiber links.
In 2011 the difference between the exact and the relaxed design approaches is less significant than in 2009. This is because in the relaxed approach, there is a need for extra fibers in some links. In this study the RWA algorithms are not aware of the limitation of 80 wavelengths per link, resulting in a non optimal usage of the fiber capacity. If the algorithms can prevent the deployment of new fibers, this would gain a lot in the advantage of the exact design algorithms.

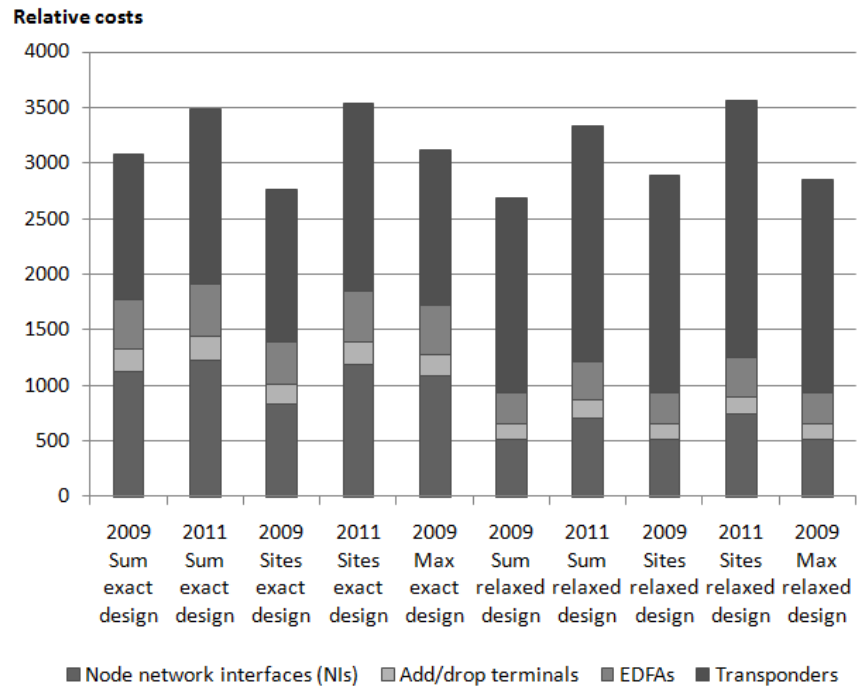

Fig. 5: Total CapEx of the different RWA algorithms, the fixed colored OXC is considered here (node 1). The greenfield infrastructure cost for 2009 and 2011 is shown

\section{2) Network related OpEx evaluation}

In Fig. 6 we can see that the network related OpEx has the same trend as the CapEx. The network OpEx of the relaxed case are relatively higher than in the exact design approach. This is caused by the higher amount of transponders, and results in a higher power consumption and repair cost. The repair cost of the active node equipment is higher in the actual case, because the increased number of WSSs. To compare the network related OpEx to the CapEx we considered a period of 5 years. Thus we multiply the network related OpEx by 5 and divide it by the total CapEx of 2011 for the sum exact strategy, and this results in $23 \%$. We can do the same exercise for the relaxed case and results in $24 \%$. If the lifetime of the investment would be higher, this ratio would be even higher. Almost a fourth of the cost of a European core network is going to network related operational expenditures. For this reason savings in network OpEx can have a large positive impact in the economic feasibility of the proposed design. In general the energy and the repair costs of the nodes are less than those for the transponders. 


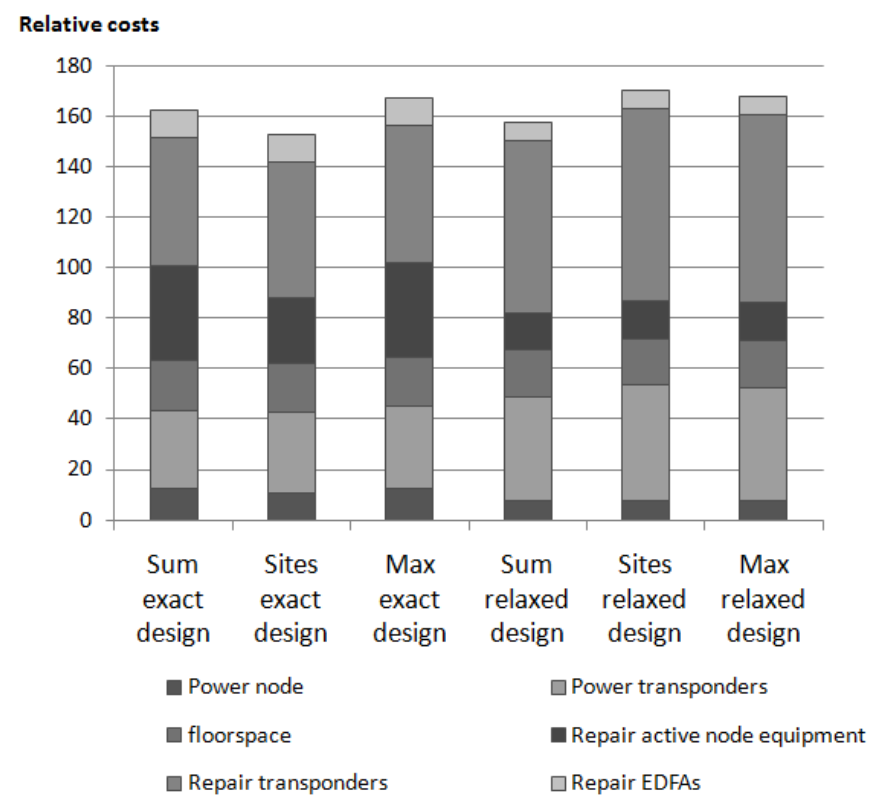

Fig. 6: The network related OpEx is shown here for the different RWA algorithms for the Greenfield rollout in 2009, the fixed colored OXC is considered here (node 1).

\section{Impact of the node architecture on the CapEx}

In Section IV we described the different node architectures. With the developed cost model we could calculate the total infrastructure cost for the different nodes. If we consider a higher flexible node, then the costs of the network interfaces and add/drop terminals increase a lot, and especially in the fully equipped node 3, see Fig. 7. In this case, it is even more interesting to limit the number of fiber links per physical link. The difference between node 2 and node 1 is $30 \%$ for the exact design approach. For the relaxed design approach it is $37 \%$. The reason is a higher number of colorless ports at the add/drop terminal. Further optimizations can be performed by reducing the number of add/drop terminals. The best case is shown with node 2 . To make use of this node, improved RWA-algorithms have to be developed to prevent wavelength blocking at the add/drop terminal. A real situation can be between total cost of node 2 and node 3 , if the RWA algorithm can prevent dropping the same wavelength more than once at one node.

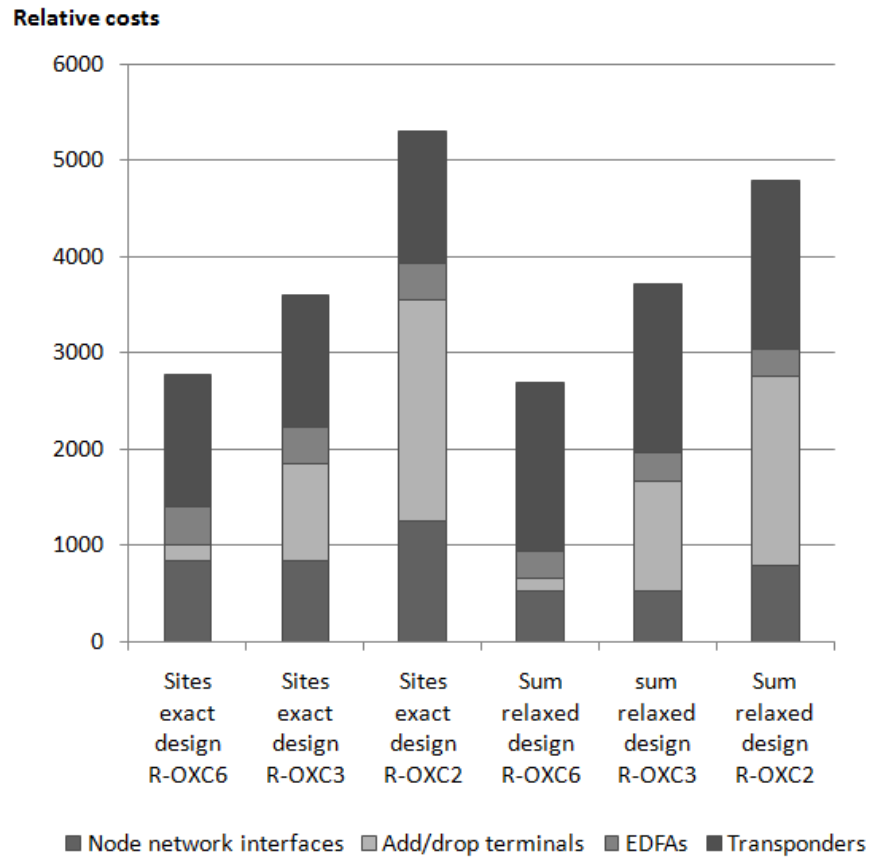

Fig. 7: The CapEx for the two best RWA algorithms (sites exact case and sum relaxed case), the three node architectures are considered here and that for a greenfield rollout in 2009

\section{CONCLUSIONS AND FUTURE WORK}

In this work we performed an in depth cost analysis of the next generation optical core networks. The impact of the Impairment Aware - Routing and wavelength assignment (IARWA) algorithms on the provisioning of regenerators in the network is also investigated. The results show that $35-48 \%$ savings on the transponders cost can be achieved when designing the network using the exact instead of the relaxed design approach. This study also suggested that it is important for the RWA optimization, to take into account the actual number of fibers in a physical link. Because if it is not possible to have the same number of fiber links in both cases it will not be possible to save costs with regenerator savings, in fact the situation can be even worse, caused of the increased number of network interfaces to connect extra fiber links. Another aspect we investigated, was the impact of the higher node flexibility to the total infrastructure cost. The total infrastructure would be two times more expensive if one considers the fully equipped OXC. Large savings can be achieved if the number of same wavelengths that have to be dropped in a node is reduced, using architecture node 2 (with non-blocking free $\mathrm{OXCs}$ ) instead of node 3 (with blocking free OXCs).

We believe that studies, such as the one performed in this work, are very important both for the economic feasibility of future optical core networks and for the realistic design of IARWA algorithms. For this reason we are interested in further extending our study in the near future, by considering bidirectional traffic and by minimizing the amount of extra fiber links and regenerators decided by the IA-RWA algorithms. Finally, we will study the impact of the node architectures on the operational costs of optical networks. Most important operational costs will be service related, like 
the cost to setup or tear down a connection. The performance indicator will be the number of manual interventions.

\section{ACKNOWLEDGMENT}

This work has been supported by the European Commission through DICONET project [6].

\section{REFERENCES}

[1] H. Zang, J. P. Jue, B. Mukherjee, "A Review of Routing and Wavelength Assignment Approaches for Wavelength-Routed Optical WDM Networks", Optical Networks Magazine, Vol. 1, 2000.

[2] J. He, M. Brandt-Pearce, Y. Pointurier, S. Subramaniam, "QoT-Aware Routing in Impairment-Constrained Optical Networks", pp. 26-30, IEEE Globecom, 2007.

[3] V. Anagnostopoulos, C. Politi, C. Matrakidis, A. Stavdas, "Physical layer impairment aware routing algorithms based on analytically calculated constraints", Optics Communications, 2006.

[4] Y. Huang, J. Heritage, B. Mukherjee, "Connection provisioning with transmission impairment consideration in optical WDM networks with high-speed channels", Lightwave Technology, Journal of, vol.23, no. 3, pp. 982-993, March 2005

[5] K. Manousakis, K. Christodoulopoulos, E. Kamitsas, I. Tomkos, E. A. Varvarigos, " Offline Impairment-Aware Routing and Wavelength Assignment Algorithms in Translucent WDM Optical Networks", J. of Lightwave Technology, Vol. 27, No. 12, pp. 1866-1877, 2009.

[6] S. Azodolmolky et al., "A dynamic impairment-aware networking solution for transparent mesh optical networks", IEEE Communications Magazine, Vol. 47, No. 5, pp. 38-47, 2009.

[7] K. Christodoulopoulos, K. Manousakis, E. A. Varvarigos, "Cross Layer Optimization of Static Lightpath Demands in Transparent WDM Optical Networks", IEEE Information Theory Workshop on Networking and Information Theory (ITW), 2009.

[8] T. Zami, A. Morea, B. Lavigne, M. Lefrançois, "Transparent Nodes. Yes, but to what extent ?", Ecoc, 2009

[9] R. Huelsermann,M. Gunkel, C. Meusburger, D.A. Schupke, "Cost modeling and evaluation of capital expenditures in optical multilayer networks", OSA Journal of Optical Networking, vol. 7, no. 9, 2008.

[10] S.Verbrugge, K. Casier, J. Van Ooteghem, B. Lannoo, "Practical steps in techno-economic evaluation of network deployment planning", IEEE Globecom, Tutorial T11, 2009.

[11] Breda in perspective, DTZ Wadelhoff Research, www.dtz.nl 